\title{
Evaluation of the Long-term Administration of Rifaximin for More than Three Years in the Treatment of Repeated and Recurrent Overt Hepatic Encephalopathy
}

\author{
Yudai Fujiwara ${ }^{1,2}$, Kazuyuki Suzuki ${ }^{1,2}$, Kenji Yusa ${ }^{1,2}$, Makoto Eizuka², Manami Miura ${ }^{2}$, \\ Yuki Watanabe ${ }^{1,2}$, Hiroshi Takahashi ${ }^{2}$ and Yasuhiro Takikawa ${ }^{1}$
}

\begin{abstract}
:
The patient was a 65-year-old man with alcoholic liver cirrhosis who had been admitted to hospital 5 times for repeated and recurrent overt hepatic encephalopathy (HE) despite numerous therapies, including disaccharide, branched-chain amino acid (BCAA) formula, L-carnitine and zinc. After the additional administration of rifaximin $(1,200 \mathrm{mg} / \mathrm{day}$ orally), his consciousness level was well controlled for 3 years without any adverse effects. The long-term administration of rifaximin may be useful and safe for managing recurrent overt HE, although the maintenance dosage and duration of rifaximin and safety should be evaluated in patients with ameliorated HE.
\end{abstract}

Key words: rifaximin, liver cirrhosis, overt hepatic encephalopathy, hyperammonemia

(Intern Med 60: 1027-1033, 2021)

(DOI: 10.2169/internalmedicine.5793-20)

\section{Introduction}

Hepatic encephalopathy (HE) is one of the most serious complications seen in patients with liver cirrhosis (LC) and is closely associated with a poor prognosis and a reduced health-related quality of life $(1,2)$. Since HE shows a wide spectrum of clinical signs, such as flapping tremor and consciousness disturbance, ranging from minor defects in orientation and coordination to deep coma, HE was recently classified into two categories: covert HE and overt HE (3). Covert $\mathrm{HE}$ involves minimal $\mathrm{HE}$ and grade I coma, while overt HE involves grade II-IV coma according to the West-Haven criteria $(1,3)$. Although the pathogenesis of $\mathrm{HE}$ in LC has not been fully clarified, neurotoxic substances, such as ammonia, mainly produced in the gut and portal-systemic shunting (PSS) are closely associated with the onset and recurrence of HE $(2,4)$. Thus, synthetic disaccharides (lactulose or lactitol) that decrease the production and absorption of ammonia in the gut have been historically used as firstline treatment for LC patients with both covert and overt HE worldwide $(2,5)$.

The oral administration of poorly absorbable antibiotics, such as neomycin, kanamycin, vancomycin, metronidazole, and polymyxin $\mathrm{B}$, has also been performed with or without synthetic disaccharides in patients with HE and/or hyperammonemia $(2,5)$. However, these drugs cannot be administered over long periods of time, as they often induce severe and irreparable complications. Furthermore, according to the guideline of the Japanese Ministry of Healthy, Labor and Welfare, these drugs are not approved for insurance coverage in medical practice.

Rifaximin is a poorly absorbable oral antimicrobial drug that has been used in many countries to manage HE and/or hyperammonemia in LC and has shown few adverse effects, even with long-term administration (6). In Japan, the administration of rifaximin in the clinical setting has been approved since December 2016 (6). Recently, two studies reported the efficacy and safety of the long-term administration of rifaximin in Japanese patients with LC $(7,8)$. However, the clinical data on the efficacy and safety of the administration of rifaximin for over two years to LC patients

${ }^{1}$ Division of Hepatology, Department of Internal Medicine, Iwate Medical University, Japan and ${ }^{2}$ Department of Gastroenterology, Iwate Prefectural Ninohe Hospital, Japan

Received for publication July 6, 2020; Accepted for publication September 10, 2020

Correspondence to Dr. Yudai Fujiwara, yudfuji@iwate-med.ac.jp 
with overt HE remain insufficient.

We herein report the case of a cirrhotic patient with repeated and recurrent overt $\mathrm{HE}$ that was well controlled by the administration of rifaximin for over three years.

\section{Case Report}

The patient was a 65-year-old man had been diagnosed with alcoholic liver injury since the beginning of May, 2004. He was admitted to the Department of Neurology in our hospital and received treatment for cerebral infarction in the right corona radiata with left numbness and weakness of the upper and lower limbs. His clinical symptoms associated with cerebral infarction completely disappeared with treatment, and he was discharged on the middle of May, 2004. However, he voluntarily stopped his medication and stopped attending follow-up appointments on the middle of November, 2011.

He was first admitted to our department on the middle of April, 2013, because of spontaneous hematemesis. Upper gastrointestinal endoscopy was immediately performed, and the focus of the bleeding was found to be esophageal varices (location, inferior to middle; form, 2; color, blue; red color sign, negative). We therefore performed endoscopic ligation therapy to treat his esophageal varices. At that time, we estimated the severity of liver damage and checked for other complications, such as hepatocellular carcinoma (HCC), with biochemistry and imaging tests [e.g., abdominal sonography and computed tomography (CT)]. The severity of liver dysfunction was Child-Pugh B, and the score was 8 points. The findings of imaging tests were incompatible with the diagnosis of LC without ascites and HCC. His estimated ethanol intake was $>52 \mathrm{~g} /$ day for 40 years. Serum virus markers related to hepatitis $\mathrm{B}$ and $\mathrm{C}$ viruses were all negative, and serum titers of antinuclear antigen and antimitochondrial antigen were also negative. Based on these findings, we diagnosed the patient with alcoholic LC without HCC. Regarding the body composition analysis, his height was $170 \mathrm{~cm}$, his body weight was $70 \mathrm{~kg}$, and his body mass index was 24.22 .

Following endoscopic treatment for esophageal varices, since no re-bleeding of the esophageal varices was noted and the liver damage was not exacerbated, he was discharged on the end of April, 2014, and followed up at the outpatient clinic of our hospital. At the first admission, he received branched-chain amino acid (BCAA) granules (12 g/ day) for hypoalbuminemia and a proton pump inhibitor (PPI; esomeprazole magnesium hydrate; $20 \mathrm{mg} /$ day). Later, as he complained abdominal distension due to ascites, diuretics (furosemide $20 \mathrm{mg} /$ day and spironolactone $50 \mathrm{mg} /$ day) were prescribed. At this point, he had completely discontinued alcohol intake since his first admission on the middle of April, 2013. However, he was again admitted to the hospital on the beginning of May, 2015, due to disturbance of consciousness. At this point, he was diagnosed with overt HE with coma grade III according to the criteria of Inuyama symposium in Japan (9).

The precipitating factors of $\mathrm{HE}$ were complex and included constipation due to decreased daily stool passage (once every two days several days before the onset of overt $\mathrm{HE}$ ), dehydration due to overdosage of diuretics and protein overload, as he mentioned consuming roughly $200 \mathrm{~g}$ of roast meat 2 days before the onset of overt HE. His laboratory data on admission showed the following: red blood cell (RBC) count, $331 \times 104 \mu \mathrm{L}$; hemoglobin $(\mathrm{Hb}), 12.6 \mathrm{~g} / \mathrm{dL}$; white blood cell (WBC) count, 3,310 $\mu \mathrm{L}$; platelet count, $60 \times$ $104 \mu \mathrm{L}$; total bilirubin (T-Bil.), $0.7 \mathrm{mg} / \mathrm{dL}$; aspartate aminotransferase (AST), $37 \mathrm{IU} / \mathrm{L}$; alanine aminotransferase (ALT), 34 IU/L; $\gamma$-glutamyl transpeptidase ( $\gamma$-GTP), 114 IU/ $\mathrm{L}$; albumin, $3.6 \mathrm{~g} / \mathrm{dL}$; prothrombin time activity (PT), 69\%; creatinine (CRNN), $1.07 \mathrm{mg} / \mathrm{dL}$; and blood ammonia (B$\mathrm{NH}_{3}$ ), $205 \mu \mathrm{g} / \mathrm{dL}$. His Child-Pugh classification was B and his Child-Pugh score was 9. Dynamic CT showed a typical LC pattern with an irregular surface and atrophic liver, with a slight amount of ascites. We immediately performed the intravenous administration of BCAA-enriched amino acid solution (Aminoleban ${ }^{\circledR}$; Otsuka Pharmaceutical, Tokyo, Japan) $500 \mathrm{~mL} /$ day with $24-\mathrm{h}$ continuous administration of a glucose-electrolyte mixed solution and then prescribed lactulose $(60 \mathrm{~mL} /$ day $)$. After these treatments, his consciousness gradually improved to a normal state. He was discharged on the middle of May, 2015, and again followed up at the outpatient clinic of our hospital with periodic evaluations (monthly). For the management of HE, he was prescribed lactulose (60 mL/day) and enteral nutrition (Aminoleban $\mathrm{EN}^{\circledR}$ : total calories: $240 \mathrm{kcal} /$ package, including $24 \mathrm{~g}$ of protein; $100 \mathrm{~g} /$ day) with a low-protein diet of $60 \mathrm{~g} /$ day, magnesium oxide (1.5 g/day) and PPI (20 mg/day).

However, he was again admitted on the beginning of July, 2015, and received treatments for overt HE (coma grade II). The precipitating factor of overt HE was constipation due to decreased daily stool passage. During this second admission period, he received kanamycin (oral, $500 \mathrm{mg}$ /day) for 14 days; however, this treatment showed no apparent effect on his consciousness level. In addition, lactulose was changed to lactitol (18 g/day), due to the fact that the patient found the lactulose to be too sweet and as a result he consumed it irregularly. Furthermore, polaprezinc (150 mg/day, including $34 \mathrm{mg} /$ day of zinc) was administered to prevent gastric mucosal injury and to improve the ammonia metabolism. Subsequently, from May 2015 to April 2017, he was admitted a total of five times because of overt HE with or without mild ascites. The final admission was the beginning of April, 2017, when he suddenly complained of disorientation early in the morning on the day of admission. The precipitating factor of HE in this instance was considered to be constipation and dehydration due to an overdose of furosemide. He immediately received infusion therapy with Aminoleban ${ }^{\circledR}$ and lactulose enema, and his coma grade improved from III to II. Although the dosage of lactitol was changed from 18 $\mathrm{g} /$ day to $36 \mathrm{~g} /$ day during admission, we decided to start rifaximin (orally 1,200 mg/day) along with other ammonia- 
Table 1. Biochemical Data before Administration of Rifaximin.

\begin{tabular}{|c|c|c|c|}
\hline Hematology & & T-Protein (g/dL) & $6.6(6.5-8.0)$ \\
\hline $\mathrm{WBC}(/ \mu \mathrm{L})$ & $2,550(3,000-8,600)^{+}$ & Albumin (g/dL) & $3.3(3.9-4.9)$ \\
\hline $\mathrm{RBC}\left(\times 10^{4} \mu \mathrm{L}\right)$ & $255(400-530)$ & $\mathrm{CRP}(\mathrm{mg} / \mathrm{dL})$ & $0.35(0-0.40)$ \\
\hline $\mathrm{Hb}(\mathrm{g} / \mathrm{dL})$ & $9.6(13.5-17.5)$ & BUN (mg/dL) & $40(8-20)$ \\
\hline $\mathrm{Ht}(\%)$ & $28.3(40.0-50.0)$ & CRNN (mg/dL) & $0.92(0.5-1.2)$ \\
\hline Platelet $\left(\times 10^{4} / \mu \mathrm{L}\right)$ & $44(130-340)$ & $\mathrm{B}-\mathrm{NH}_{3}(\mu \mathrm{g} / \mathrm{mL})$ & $140(30-86)$ \\
\hline Blood Chemistry & & BTR & not tested \\
\hline T-Bil. (mg/dL) & $0.7(0.2-1.2)$ & Blood coagulation & \\
\hline AST (IU/L) & $87(8-40)$ & PT activity/PT-INR & $52 \% / 1.40$ \\
\hline ALT (IU/L) & $35(5-35)$ & Tumor markers & \\
\hline ALP (IU/L) & $292(112-334)$ & $\mathrm{AFP}(\mathrm{ng} / \mathrm{mL})$ & $3.0(0.0-13.4)$ \\
\hline$\gamma \mathrm{GTP}(\mathrm{IU} / \mathrm{L})$ & $66(8-65)$ & PIVKA-II (mAU/mL) & $41(0-39)$ \\
\hline \multicolumn{4}{|c|}{ 3iochemical examination was performed in April, 2017.} \\
\hline \multicolumn{4}{|c|}{$\begin{array}{l}\text { WBC: white blood cells, RBC: red blood cells, Hb: hemoglobin, Ht: hematocrit, T-Bil.: total bilirubin, } \\
\text { AST: aspartate aminotransferase, ALT: alanine aminotransferase, ALP: alkaline phosphatase, } \gamma \mathrm{GTP} \text { : } \\
\gamma \text {-glutamyl transpeptidase, T-Protein: total protein, CRP: C-reactive protein, BUN: blood urea nitro- } \\
\text { gen, CRNN: creatinine, B-NH3: plasma ammonia concentration, BTR: serum branched-chain amino } \\
\text { acids to tyrosine ratio, PT-INR: prothrombin time-international ratio, anti-mitochondrial antibody, } \\
\text { AFP: } \alpha \text {-fetoprotein, PIVKA-II: prothrombin-induced vitamin K absent-II }\end{array}$} \\
\hline
\end{tabular}

lowering agents from the end of April, 2017.

Table 1 shows the laboratory data obtained before the administration of rifaximin, including pancytopenia, hypoalbuminemia, low prothrombin time-international normalized ratio, and hyperammonemia. The Child-Pugh score was 9 points. Tumor markers for HCC were almost within the baseline range. Abdominal CT showed an atrophic liver without HCC or ascites (small volume) with marked splenomegaly. Furthermore, on the day of rifaximin administration, we performed a number connection test (NCT) A, which is a neuropsychiatric test to estimate the coma grade in cases of HE, and the time was over 120 seconds (normal baseline $<30$ seconds and time-over $>120$ seconds) (10).

The serial changes in the $\mathrm{B}-\mathrm{NH}_{3}$ and serum albumin levels after the administration of rifaximin with other medications are shown in Figure A. The overall B- $\mathrm{NH}_{3}$ levels after the administration of rifaximin were $62 \mu \mathrm{g} / \mathrm{dL}$ at 1 month, $112 \mu \mathrm{g} / \mathrm{dL}$ at 3 months, $43 \mu \mathrm{g} / \mathrm{dL}$ at 6 months, $33 \mu \mathrm{g} / \mathrm{dL}$ at 12 months, $54 \mu \mathrm{g} / \mathrm{dL}$ at 24 months and $72 \mu \mathrm{g} / \mathrm{dL}$ at 36 months. Interestingly, the serum albumin level was maintained after the administration of rifaximin despite stopping and/or decreasing BCAA granules and BCAA-enriched nutrient administration. The changes in the Child-Pugh score and serum creatinine level are shown in Figure B. These parameters were not exacerbated during the course of rifaximin administration. The serum zinc level was almost in the normal range ( $\geq 60 \mu \mathrm{g} / \mathrm{dL})$, although this value was irregularly measured during rifaximin administration. Among the treatments, because the serum albumin levels showed a slight decrease in March 2018 and the $\mathrm{B}^{-\mathrm{NH}_{3}}$ level transiently increased in September 2019, BCAA granules (12 g/ day) and L-carnitine (300 mg/day) were re-administered. Furosemide was stopped, and the minimum dosage of spironolactone was administered after rifaximin administration
(Table 2). Finally, overt HE was completely prevented during the three-year period after the administration of rifaximin, although we did not conduct detailed neuropsychiatric tests to diagnose minimal HE. Furthermore, no apparent or severe adverse reactions due to the administration of rifaximin were recognized during the three-year period after the administration of rifaximin.

During the follow-up period, the patient underwent endoscopic mucosal resection for early gastric cancer $(10 \mathrm{~mm}$ in diameter at the angle of the stomach, type IIc+IIa according to the endoscopic criteria Japan Society of Gastroenterology) on the middle of March, 2019. Furthermore, he received radiofrequency ablation therapy for a small HCC (16 $\mathrm{mm}$ in diameter in segment 5 of the liver) without any marked increase in serum tumor marker levels that was detected during a routine follow-up examination via abdominal sonography and dynamic CT in March 2020. These therapies for early gastric cancer and HCC were able to be performed safely without the exacerbation of $\mathrm{HE}$ or any other complications, such as infection or an exacerbation of the patient's liver damage. At present, in May 2020, his consciousness level is almost normal, and his general condition is relatively well-maintained.

\section{Discussion}

A systemic evaluation, including an assessment of the severity of liver damage, the existence of PSS, participating factors, exacerbating factors and other complications [e.g., spontaneous bacterial peritonitis (SBP)], should be performed prior to the management of $\mathrm{HE}$ in LC patients $(1,2,11)$ In particular, an evaluation of the disease type according to the severity of liver damage is very important; for example, hepatocellular injury-dominant type (in- 


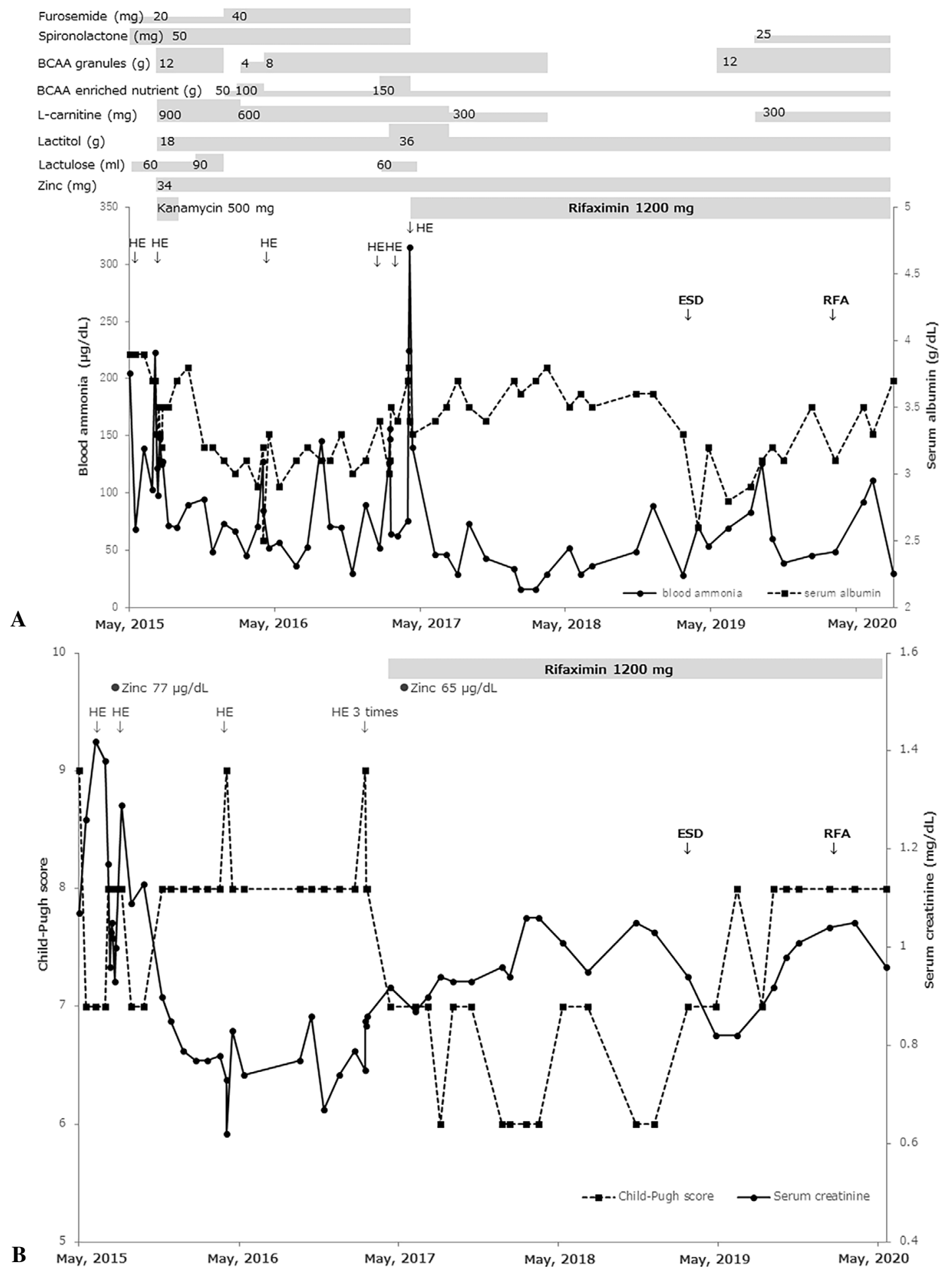

Figure. A, B: The clinical course before and after the administration of rifaximin. BCAA: Branchedchain amino acids, HE: hepatic encephalopathy, ESD: endoscopic submucosal dissection, RFA: radiofrequency ablation therapy, HCC: hepatocellular carcinoma

cluding acute-on-chronic type) usually shows hyperbilirubinemia and low prothrombin time activity, while shuntdominant type shows slight or mild hepatocellular damage. These disease types directly affect the prognosis and quality of life (11). Our case showed Child-Pugh grade B liver damage before the administration of rifaximin, which was maintained at grade $\mathrm{B}$ throughout the administration of ri- faximin. However, regarding PSS, although only esophageal varices were observed at the first admission (2015), dilation of the inferior mesenteric vein from the superior mesenteric vein and veins of retzius from the inferior vena cava was newly observed at the fifth admission (2017). No other shunts, including splenorenal shunt, were observed during the course of illness. 
Table 2. History of Admission and Medications for Hepatic Encephalopathy before and after Administration of Rifaximin.

\begin{tabular}{|c|c|}
\hline Before administration of rifaximin (2015.5-2017.4) & After administration of rifaximin (2017.4-2020.5) \\
\hline History of admission/maximal coma grade of $\mathrm{HE}$ & History of admission/maximal coma grade $\mathrm{f} \mathrm{HE}$ \\
\hline First admission: $2015.5 / \mathrm{III}$ & No newly admission. \\
\hline \multicolumn{2}{|l|}{ Second admission: $2015.7 / \mathrm{II}$} \\
\hline \multicolumn{2}{|l|}{ Third admission: $2016.4 / \mathrm{II}$} \\
\hline \multicolumn{2}{|l|}{ Fourth admission: 2017.2/II } \\
\hline \multicolumn{2}{|l|}{$\begin{array}{l}\text { Fifth admission: 2017.4/III (involves the period of administration of } \\
\text { rifaximin) }\end{array}$} \\
\hline Medications for HE (including ammonia-lowering agents) & Medications (including ammonia-lowering agents) \\
\hline Lactulose (60 mL $\rightarrow 90 \mathrm{~mL} /$ day) changes to lactitol (18 g/day $\rightarrow 36 \mathrm{~g} /$ day $)$ & Lactitol (36 g/day $\rightarrow 18$ g/day) \\
\hline Kanamycin sulfate ( $500 \mathrm{mg} /$ day, only 2 weeks in first admission only) & Rifaximin $(1,200 \mathrm{mg} /$ day $)$ \\
\hline BCAA enriched nutrient (Aminoleban $\mathrm{EN} 囚)$ & BCAA enriched nutrient (Aminoleban $\left.\mathrm{EN}^{\circledR}\right)(100 \mathrm{~g} / \mathrm{day} \rightarrow 50 \mathrm{~g} /$ day $)$ \\
\hline $50 \mathrm{~g} /$ day $\rightarrow 100 \mathrm{~g} /$ day $\rightarrow 150 \mathrm{~g} /$ day $\rightarrow 100 \mathrm{~g} /$ day & BCAA granules (LIVACT ${ }^{\circledR}$ granules) $(8 \mathrm{~g} /$ day $\rightarrow$ stopped $\rightarrow 12 \mathrm{~g} /$ day $)$ \\
\hline BCAA granules (LIVACT ${ }^{\circledR}$ granules) $(12 \mathrm{~g} /$ day $\rightarrow 8 \mathrm{~g} /$ day $)$ & L-carnitine $(900 \mathrm{mg} /$ day $\rightarrow 600 \mathrm{mg} /$ day $\rightarrow 300 \mathrm{mg} / \mathrm{day} \rightarrow$ stopped \\
\hline L-carnitine $(300 \mathrm{mg} /$ day $\rightarrow 600 \mathrm{mg} /$ day $\rightarrow 900 \mathrm{mg} /$ day $)$ & $\rightarrow 300 \mathrm{mg} /$ day $\rightarrow 600 \mathrm{mg} /$ day $)$ \\
\hline Zinc (polaprezinc) (34 mg/day) & Zinc (polaprezinc) (34 mg/day) \\
\hline Other medications & Other medications \\
\hline Proton pump inhibitor (esomeprazole magnesium hydrate) $20 \mathrm{mg} /$ day & Proton pump inhibitor (esomeprazole magnesium hydrate) $20 \mathrm{mg} /$ day \\
\hline Magnesium oxide ( $1.5 \mathrm{~g} /$ day $)$ with other laxatives & Magnesium oxide (0.7-15 g/day) with other laxatives \\
\hline Clostridium butyricum (Miya BM $\left.{ }^{\circledR}\right)(3.0 \mathrm{~g} /$ day $)$ & Clostridium butyricum (Miya BM $\left.{ }^{\circledR}\right)(3.0 \mathrm{~g} /$ day $)$ \\
\hline Diuretics (furosemide $20 \mathrm{mg} /$ day and spironolactone $50 \mathrm{mg} /$ day) & Diuretics (stopped $\rightarrow$ spironolactone 25 mg/day) \\
\hline
\end{tabular}

HE: hepatic encephalopathy, BCAA: branched-chain amino acid

Precipitating factors of HE in LC include dietary protein overload, gastrointestinal bleeding (rupture of gastroesophageal varices), bowel movement abnormality (mainly constipation), infection, excessive administration of sedative/ analgesic agents, electrolyte abnormality due to overdose of diuretic agent, dehydration, and surgery, including transjugular intrahepatic portosystemic shunt. Furthermore, hypoxemia, systemic circulatory disturbance, hypoglycemia, hypotension, serum electrolyte abnormality (particularly sodium, potassium and magnesium), and hypoalbuminemia have been also known to exacerbate HE (11). In the present case, constipation and/or the over-intake of protein might have had an important impact on the patient's condition, despite the administration of lactitol and magnesium oxide, lubiprostone and sennoside A.B calcium. In addition, dehydration due to the overdose of furosemide might have precipitated the onset of overt HE at the fifth admission before the administration of rifaximin.

Lactulose or lactitol is a first-line therapy in LC patients with overt HE and/or hyperammonemia. In Japan, BCAAenriched formulas, including infusion solutions, enteral nutritional supplement, and BCAA granules, have been also widely used, depending on both the clinical stage (coma or recovery stage) and the presence or absence of proteinenergy malnutrition (PEM) (11-14). Infusion therapy with BCAA-enriched amino acid solution has generally been used during the overt coma stage, although its use has not been adopted worldwide $(11,12)$. Enteral nutritional supplement (Aminoleban $\mathrm{EN}^{\circledR}$ ) and BCAA granules have been used in the recovery stage of $\mathrm{HE}$ and/or with PEM $(11,13,14)$. In particular, it is necessary to adjust the amount of enteral nutritional supplement according to the dietary protein dosage. Furthermore, L-carnitine (L-CA) and zinc have been used as ammonia-lowering agents. Carnitine (CA) plays an important role in fat metabolism and energy production in the mitochondria and is also closely associated with the detoxification of ammonia via the urea cycle (15). LC is a disease that shows a secondary state of CA deficiency (16), although our recent study showed that the prevalence of secondary CA deficiency is low in LC, as evaluated by the serum CA level (17). At present, although the administration of L-CA and/or acetyl-L-CA (ALC) has also been used as an optional therapy for LC patients with overt or covert HE (18), this drug is still not covered by the Japanese National Insurance System when used to treat overt HE (19). Zinc is an essential trace element with various biological effects (20). As a previous report suggests that zinc deficiency is closely associated with hyperammonemia (21), zinc supplementation has been evaluated in LC patients with hyperammonemia and/or HE (22). Our recent study of Japanese LC patients with hyperammonemia also showed that zinc (zinc acetate) supplementation for three months seemed effective and safe (23). Furthermore, we clearly indicated that the serum zinc level in LC patients showed a negative correlation with the serum albumin level (24). Although these data suggest that zinc supplementation may be effective for managing hyperammonemia, zinc acetate and other drugs containing zinc (e.g., polaprezinc used in this case), the use of these drugs in the treatment of overt $\mathrm{HE}$, is not currently approved for insurance coverage.

Table 2 summarizes the history of admissions and medications in our case before and after the administration of rifaximin. The $\mathrm{B}-\mathrm{NH}_{3}$ level stabilized after the initiation of rifaximin treatment, which was effective for reducing the 
doses of other drugs in the long-term management of HE.

Rifaximin is a second-line drug for the treatment of $\mathrm{HE}$, and its indications-according to the guidelines of the American Association for the Study of Liver Diseases and European Association for the Study of the Liver-include HE (covert HE and overt HE), idiopathic PSS, and a hyperammonemic state in chronic liver disease (1-3). Rifaximin is also recommended for use in LC patients with overt HE and/or hyperammonemia by the Japanese Society of Liver Diseases (19). However, because data on Japanese LC patients with HE (coma grade I) and/or hyperammonemia are limited to within three months, the efficacy and safety of long-term rifaximin treatment have remained unclear (6). Recently, Nishida et al. reported that long-term rifaximin treatment (median follow-up period: 62 weeks) was effective and safe in decompensated LC patients with overt HE (7). Furthermore, Suzuki et al. also showed that long-term treatment with rifaximin (median follow-up period: 41.6 weeks) was beneficial for HE without the exacerbation of the patient's liver damage and that it reduced the recurrence rate in $\mathrm{HE}$ patients without ascites (8). In our case, rifaximin was administered for more than three years and prevented repeated and recurrent overt HE. In addition, no severe adverse drug reactions, such as an exacerbation of the patient's liver damage or the onset of a Clostridium difficile infection, which are usually closely related to the long-term administration of rifaximin, were observed.

Recently, the administration of rifaximin has been shown to ameliorate changes in the composition and functionality of the gut microbiota in LC patients with HE (25). Our previous study suggests that rifaximin alters gut microbiota components associated with the neuropsychological function (26). Furthermore, rifaximin significantly reduced the level of endotoxin in the blood without affecting the composition of the gut microbiome in LC patients with HE (27).

The cost-effectiveness of rifaximin treatment in patients with HE has been also shown by Jesudian et al., who suggested that rifaximin plus lactulose was advantageous in comparison to lactulose monotherapy with regard to costbenefit, and that rifaximin maintenance treatment is very important for patients with at risk for the development of recurrent HE (28).

Regarding the dosage of rifaximin, Khokhar et al. found no significant difference in the efficacy of rifaximin (550 $\mathrm{mg}$ ) once daily and twice daily $(550 \mathrm{mg} /$ day and 1,100 mg/ day, respectively) in the prevention of HE (29). In contrast, however, Sarwar et al. reported that the rate of HE development during 6 months of follow-up was similar between a low-dose (400 mg/day) group and a full-dose group (1,100 $\mathrm{mg}$ /day) (30). Thus, the ideal duration and dose of rifaximin for the prevention of recurrent HE needs to be evaluated and elucidated in the near future.

The therapeutic effects of rifaximin and ammonialowering therapy on minimal HE and muscle atrophy (sarcopenia) have also been investigated, and the effectiveness of such an approach has been reported (31). However, in the present case, as we did not perform detailed neuropsychiatric tests (10) and did not examine the changes in muscle atrophy using $\mathrm{CT}$, we were unable to clarify whether or not the administration of rifaximin with ammonia-lowering therapy could completely prevent even minimal HE and the progression of muscle atrophy after the amelioration of HE.

Rifaximin has also been administered to LC patients with SBP (32). SBP is a severe complication in LC patients and is closely associated with the prognosis of such patients. In our case, although slight or mild ascites was often observed during the course of the illness, no apparent SBP was evident. The long-term administration of rifaximin might prevent the development of SBP.

Several intrahepatic and extrahepatic complications appear during long-term treatment in LC patients. Indeed, the present case was complicated with early gastric cancer and HCC. It was considered that the long-tern control of overt HE by treatments involving rifaximin therapy could allow these complications to be smoothly treated.

In conclusion, we experienced a cirrhotic patient with repeated and recurrent overt HE that was could be well controlled by the administration of rifaximin for more than three years. Although no apparent adverse drug reactions were observed in this case, in the near future, further studies and discussions concerning the efficacy and safety of the long-term administration of rifaximin are needed to confirm the ideal dosage and duration of rifaximin treatment for cirrhotic patients with ameliorated $\mathrm{HE}$.

Informed consent was obtained from the patient included in this study.

\section{The authors state that they have no Conflict of Interest (COI).}

Yudai Fujiwara and Kazuyuki Suzuki contributed equally to this work.

\section{References}

1. Ferenci P, Lockwood A, Mullen K, Tarter R, Weissenborn K, Blei AT. Hepatic encephalopathy - definition, nomenclature, diagnosis, and quantification. Hepatology 35: 716-721, 2002.

2. Atluri DK, Prakash R, Mullen KD. Pathogenesis, diagnosis, and treatment of hepatic encephalopathy. J Clin Exp Hepatol 1: 77-86, 2011.

3. Vilstrup H, Amodio P, Bajaj J, et al. Hepatic encephalopathy in chronic liver disease: 2014 Practice Guideline by the American Association for the Study of Liver Diseases and the European Association for the Study of the Liver. Hepatology 60: 715-735, 2014.

4. Conn HO, Leevy CM, Vlahcevic ZR, et al. Comparison of lactulose and neomycin in the treatment of chronic portal systemic encephalopathy. A double blind controlled trial. Gastroenterology 72: 573-583, 1977.

5. De Marco F, Santamaria AP, D'arienzo A. Rifaximin in collateral treatment of portal-systemic encephalopathy: a preliminary report. Current Ther Res 36: 668-674, 1984.

6. Suzuki K, Endo R, Takikawa Y, et al. Efficacy and safety of rifaximin in Japanese patients with hepatic encephalopathy: a phase 
II/III, multicenter, randomized, evaluated-blinded, active-controlled trial and a phase III, multicenter, open trial. Hepatol Res 48: 411423, 2018.

7. Nishida S, Hamada K, Nishino N, et al. Efficacy of long-term rifaximin treatment for hepatic encephalopathy in the Japanese. World J Hepatol 27: 531-541, 2019.

8. Suzuki H, Sezaki H, Suzuki F, et al. Real-world effects of longterm rifaximin treatment for Japanese patients with hepatic encephalopathy. Hepatol Res 49: 1406-1413, 2019.

9. Mochida S, Takikawa Y, Nakayama N, et al. Classification of the etiologies of acute liver failure in Japan: a report by the Intractable Hepato-Biliary Diseases Study Group of Japan. Hepatol Res 44: 365-367, 2014.

10. Kato A, Kato M, Ishii H, et al. Development of quantitative neuropsychological tests for diagnosis of subclinical hepatic encephalopathy in liver cirrhosis and establishment of diagnostic criteria multicenter collaborative study in Japan. Hepatol Res 30: 71-78, 2004.

11. Suzuki K, Kato A, Takikawa Y. Therapeutic strategies and current managements for hepatic encephalopathy. OBM Hepatol Gastroenterol 3: 18, 2019.

12. Fischer JE, Rosen HM, Ebeid AM, et al. The effect of normalization of plasma amino acids on hepatic encephalopathy. Surgery 80: 77-91, 1975.

13. Suzuki K, Kato A, Iwai M. Branched-chain amino acid treatment in patients with liver cirrhosis. Hepatol Res 30S: S25-S29, 2004

14. Muto Y, Sato S, Watanabe A, et al. Effects of oral branched chain amino acid granules on event-free survival in patients with liver cirrhosis. Clin Gastroenterol Hepatol 3: 705-713, 2005.

15. Vaz FM, Wanders RJ. Carnitine biosynthesis in mammals. Biochem J 361: 417-429, 2002.

16. Rudman D, Sewell CW, Ansley JD. Deficiency of carnitine in cachectic cirrhotic patients. J Clin Invest 60: 716-723, 1977.

17. Suzuki K, Onodera M, Kuroda H, et al. Reevaluation of serum carnitine status in patients with liver cirrhosis. J Liver Res Disord Ther 2: 00025, 2016.

18. Malaguarnera M, Pistone G, Elvira R, Leotta C, Scarpello L, Liborio R. Effects of L-carnitine in patients with hepatic encephalopathy. World J Gastroenterol 11: 7197-7202, 2005.

19. Fukui H, Saito H, Ueno Y, et al. Evidence-based clinical practice guidelines for liver cirrhosis. J Gastroenterol 51: 629-650, 2016.

20. Stamoulis I, Kouraklis G, Theocharis S. Zinc and the liver: an active interaction. Dig Dis Sci 52: 1595-1612, 2007.

21. Van der Rijt CC, Schalm SW, Schat H, Foeken K, De Jong G. Overt hepatic encephalopathy precipitated by zinc deficiency. Gas- troenterology 1004: 1114-1118, 1991.

22. Riggio O, Ariosto F, Merli M, et al. Short-term oral zinc supplementation does not improve chronic hepatic encephalopathy. Results of as double-blind crossover trial. Did Dis Sci 36: 12041208, 1991.

23. Katayama K, Saito M, Kawaguchi T, et al. Effect of zinc on liver cirrhosis with hyperammonemia: a preliminary randomized, placebo-controlled double-blind trial. Nutrition 30: 1409-1414, 2014.

24. Katayama K, Kawaguchi T, Shiraishi K, et al. The prevalence and implication of zinc deficiency in patients with chronic liver disease. J Clin Med Res 10: 437-444, 2018.

25. Riordan SM, Williams R. Gut flora and hepatic encephalopathy in patients with cirrhosis. N Engl J Med 362: 1140-1142, 2010.

26. Kawaguchi T, Suzuki F, Imamura M, et al. Rifaximin-altered gut microbiota components associated with liver/neuropsychological functions in patients with hepatic encephalopathy: An exploratory data analysis of phase II/III clinical trial. Hepatol Res 49: 404418, 2019.

27. Kaji K, Takaya H, Saikawa S, et al. Rifaximin ameliorates hepatic encephalopathy and endotoxemia without affecting the gut microbiome diversity. World J Gastroenterol 23: 8355-8366, 2017.

28. Jesudian AB, Ahmad M, Bozkaya D, et al. Cost-effectiveness of rifaximin treatment in patients with hepatic encephalopathy. JMCP 26: 750-756, 2020.

29. Khokhar N, Qureshi MO, Ahmad S, et al. Comparison of once day rifaximin to twice a day dosage in the prevention of recurrence of hepatic encephalopathy in patients with chronic liver disease. J Gastroenterol Hepatol 30: 1420-1422, 2015.

30. Sarwar S, Muhyuddin B, Aleem A, Nadeem MA. Primary prophylaxis of hepatic encephalopathy in decompensated cirrhosis: low dose vs. full dose rifaximin. Pak J Med Sci 35: 1446-1450, 2019.

31. Bajaj JS, Heuman DM, Wade JB, et al. Rifaximin improves driving simulator performance in a randomized trial of patients with minimal hepatic encephalopathy. Gastroenterology 140: 478-487, 2011.

32. Hanouneh MA, Hanouneh IA, Hashash JG, et al. The role of rifaximin in the primary prophylaxis of spontaneous bacterial peritonitis in patients with liver cirrhosis. J Clin Gastroenterol 46: 709-717, 2012

The Internal Medicine is an Open Access journal distributed under the Creative Commons Attribution-NonCommercial-NoDerivatives 4.0 International License. To view the details of this license, please visit (https://creativecommons.org/licenses/ by-nc-nd/4.0/).

(C) 2021 The Japanese Society of Internal Medicine Intern Med 60: 1027-1033, 2021 